\title{
OPIOID RECEPTOR MRNA EXPRESSION IN PRIMARY CULTURES OF GLIAL CELLS DERIVED FROM DIFFERENT RAT BRAIN REGIONS
}

Bianca B. Ruzicka, Charles A. Fox, Robert C. Thompson, Huda Akil and Stanley J. Watson, Mental Health Research Institute, University of Michigan, 205 Zina Pitcher Place, Ann Arbor, MI, 48109-0720.

Glial cell cultures derived from the cortex (Ctx), striatum (Str), cerebellum (Cb), hippocampus (Hippo) or hypothalamus (Hypo) were analyzed with respect to opioid receptor mRNA expression using ribonuclease protection assays. Results of this study suggest that glial cells possess mu $(\mu)$, delta $(\delta)$ and kappa $(\kappa)$ receptors in a type- and brain region-dependent manner.

Opioid receptors, generally classified as $\mu, \delta$ or $\kappa$, are widely distributed throughout the brain and spinal cord. The activation of these receptors results in several physiological responses, including analgesia and tolerance/dependence. Although historically these receptors have been presumed to have a neuronal localization, recent biochemical studies have suggested that these proteins may also be associated with glial cell membranes [1, 2, 3]. However, radioligand binding studies have yielded equivocal results $[4,5]$. The recent cloning of the $\mu, \delta$ and $\kappa$ receptors has enabled us to directly examine the issue of opioid receptor mRNA expression in primary cultures of rat brain astroglia. In this regard, two questions were addressed in this study: first, for an individual brain region, what is the relative glial expression profile for the different opioid receptor types? Second, for each opioid receptor type, what is its glial distribution profile across different brain regions? To address these questions, the $\mathrm{Ctx}$, Str, $\mathrm{Cb}$, Hippo and Hypo from postnatal day 1 rats were dissected and processed by trypsinization and gradient centrifugation to obtain a mixed neuronal-glial cell suspension. Cells, seeded at $5 x$ $10^{5 / 10} \mathrm{~cm}$ culture plate, were grown for 13 days, until $80-90 \%$ confluent, under conditions favoring astrocyte proliferation. The cells were then lysed directly on the culture plates with Trizol ${ }^{\circledR}$ Reagent (Gibco-BRL, Long Island, NY) to extract total RNA. The RNA was analyzed for $\mu, \delta$ and $\kappa$ mRNA using ribonuclease protection assays. Autoradiogram signals generated from protected [32 P]-labeled RNA fragments were analyzed with respect to the intensity of gray level using computerized image analysis (NIH Image). The results of preliminary studies are presented in summarized form in figure 1 .

Fig. 1: Relative Abundance of Opioid Receptor mRNA Expression in Primary Glial Cell Cultures
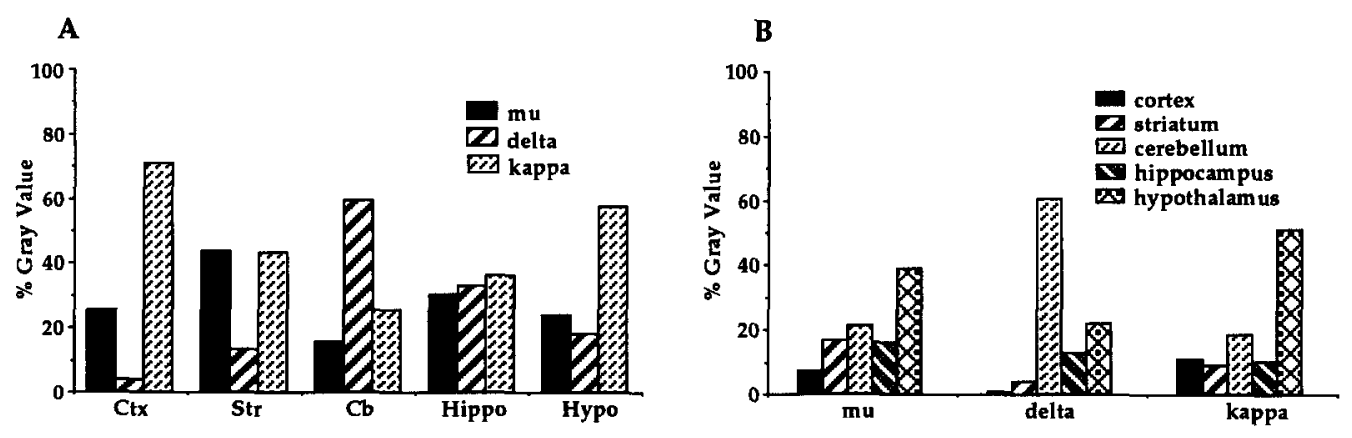
The results show that primary cultures of glial cells derived from different brain regions expressed opioid receptor mRNA. In this regard, the hypothalamic and cerebellar cultures expressed the highest levels of total opioid receptor mRNA, which represented an approximately 3-fold greater expression level than that observed in glial cultures derived from the Ctx, Str or Hippo (data not shown). Moreover, regarding the total $\mu, \delta$ and $\kappa$ mRNA expression across different glial cultures, the apparent rank order of abundance was $\kappa>\delta>\mu$ (data not shown). Glial cell cultures derived from different brain regions were characterized by unique relative opioid receptor mRNA expression profiles (fig. 1A). Cultures derived from the Hypo and $\mathrm{Ctx}$ were characterized by an abundance of $\mathrm{K}$ mRNA. In contrast, cerebellar cultures were characterized by an abundance of $\delta \mathrm{mRNA}$. The striatal and hippocampal cultures also possessed distinct relative expression profiles for the opioid receptors: cultures derived from the Str exhibited equal $\mu$ and $\kappa$, but low $\delta$, mRNA expression levels, while cultures derived from the Hippo displayed similar $\mu, \delta$ and $\kappa$ mRNA expression levels. The glial distribution profiles for the opioid receptors across different brain regions varied with the receptor type (fig. 1B). The expression of $\mu$ mRNA was highest in glial cell cultures derived from the Hypo, moderate in the striatal, cerebellar and hippocampal cultures, and relatively low in the cortical cultures. In contrast, the distribution profiles for the $\delta$ and $\kappa$ mRNAs were characterized by apparent rank orders of abundance of $\mathrm{Cb}>\mathrm{Hypo}>$ Hippo $>\mathrm{Str}>$ $\mathrm{Ctx}$ and $\mathrm{Hypo}>>\mathrm{Cb}>\mathrm{Ctx} \geq \mathrm{Str}=\mathrm{Hippo}$, respectively. Glial cells, and not neurons, were the apparent source of the opioid receptor mRNA expression, as supported by the observation that no neurons were detected by immunocytochemical analysis for neuron specific enolase (NSE) in cortical, striatal, cerebellar and hippocampal cultures. In hypothalamic cultures, 6 cells of approximately 100000 cultured cells were NSE positive. These data raise the question of relative glial versus neuronal opioid receptor mRNA expression levels. Although the answer to this question is not presently known, preliminary quantitative analyses suggest that these expression levels may differ as much as 55-fold, depending on the receptor type and the brain region. For example, cerebellar glial cell cultures appear to express 40 -fold more $\kappa$ mRNA than fresh, homogenized cerebellar tissue. In contrast, fresh, homogenized cortical tissue appears to express 55 -fold more $\delta$ mRNA than the cortical glial cell cultures. These data also raise the question of the physiological significance of the glial localization of opioid receptors. Although this is presently unclear, these receptors may play a role in those conditions of neuronal hyperactivity, trauma and/or degeneration associated with gliosis.

\section{Acknowledgements}

This work was supported by the Medical Research Council of Canada, NIDA (grant DA 02265) and the Lucille P. Markey Charitable Trust (\#88-46).

\section{References}

1. G. Rougon et al, Nature 305: 715, 1983.

2. B. Pearce et al, Neuroscience Lett. 55: 157, 1985.

3. P.S. Eriksson et al, Neurochem. Res. 15: 1123, 1990.

4. C.M. Hendrickson and S. Lin, Neuropharmacology 19: 731, 1980.

5. K. Maderspach and R. Solomania, Brain Res. 441: 41, 1988. 